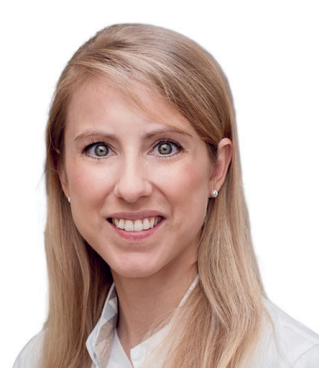

\title{
Depressive Psoriasis-Patienten können ein schlechteres Ansprechen auf Etanercept zeigen
}

Wiebke Sondermann

Universitätsklinikum Essen, Klinik für Dermatologie, Venerologie und Allergologie, Essen, Deutschland

Abstract aus Jin W, Zhang S, Duan Y: Depression symptoms predict worse clinical response to etanercept treatment in psoriasis patients. Dermatology 2019;235:55-64.

\author{
Keywords \\ Psoriasis · Depression · Anxiety · Etanercept · Response
}

\section{Abstract}

Background/Aims: This study aimed to investigate the predicting values of depression and anxiety symptoms for clinical response to etanercept treatment in psoriasis patients.

Methods: A total of 85 psoriasis patients who received 6 months of etanercept treatment were consecutively enrolled in this prospective cohort study. The Psoriasis Area and Severity Index (PASI) score was evaluated at month 0 (M0), M1, M3, and M6, and the corresponding PASI 75/90 response at each visit was assessed. Also, anxiety and depression symptoms were assessed by the Hospital Anxiety and Depression Scale (HADS) at M0, M1, M3, and M6.

Results: Depression symptoms were observed to correlate with female gender ( $p=0.004)$, longer disease duration $(p=0.018)$, and higher PASI score $(p<0.001)$, and anxiety symptoms were seen to be associated with female gender $(p=0.017)$, larger psoriasis-affected body surface area $(p=0.049)$, and higher PASI score $(p=$ 0.017 ) in psoriasis patients. After etanercept treatment, HADS-Depression (HADS-D) and HADS-Anxiety (HADS-A) scores were both decreased at M1, M3, and M6 (all $p<0.001$ ) compared with M0. Most importantly, baseline depressed patients presented with a lower PASI 75 response rate at M3 $(p=0.014)$ and M6 $(p=0.005)$, and a reduced PASI 90 response rate at M6 ( $p=0.045)$ compared with baseline non-depressed patients. Furthermore, multivariate logistic regression analyses revealed that depression symptoms at baseline were an independent predictive factor for the lower possibility of both PASI 75 response $(p=0.048)$ and PASI 90 response $(p=0.048)$ achievements at M6 in psoriasis patients. However, no correlation of baseline anxiety symptoms with PASI 75/90 responses was observed.

Conclusion: Depression symptoms at baseline independently predict a worse clinical response to etanercept treatment in psoriasis patients.

C 2018 S. Karger AG, Basel

\section{KARGER}

(C) 2019 S. Karger GmbH, Freiburg 


\section{Transfer in die Praxis}

\section{Hintergrund}

In den letzten Jahren konnte immer besser herausgearbeitet werden, dass Patienten mit Psoriasis überdurchschnittlich häufig unter psychischen Komorbiditäten wie Depressionen, Suchterkrankungen und Angststörungen leiden. Auch haben Psoriatiker, vor allem schwer betroffene Patienten, eine erhöhte Suizidalitätsrate im Vergleich zu Hautgesunden [1].

Je nach Studiendesign, untersuchter Population und den zugrunde gelegten diagnostischen Kriterien geben bis zu 62\% der Psoriasis-Patienten depressive Symptome an und bis zu 43\% der Patienten berichten von Angstgefühlen [2, 3].

Die erhöhte Depressionsprävalenz bei Psoriasis ist jedoch nicht allein als Reaktion auf die Erkrankung zu sehen, sondern besteht zum Teil auch unabhängig vom Schweregrad der Psoriasis. Es gibt mittlerweile eine gute Evidenz dafür, dass der Psoriasis und Depressionserkrankungen überlappende biologische Mechanismen zugrunde liegen. So finden sich bei beiden Erkrankungen erhöhte Konzentrationen proinflammatorischer Zytokine, was darauf hinweist, dass der systemische Entzündungsprozess bei beiden Erkrankungen eine wichtige Rolle spielt [1, 4].

Psychische Faktoren können einen Einfluss auf das therapeutische Ansprechen verschiedener Dermatosen haben $[5,6]$.

Das Ziel der vorliegenden Studie war es, näher zu beleuchten, inwiefern Depressionen und Ängstlichkeit/Angststörungen das therapeutische Ansprechen von Psoriasis-Patienten auf Etanercept beeinflussen.

\section{Ergebnisse}

Insgesamt wurden 85 Psoriasis-Patienten, die über 6 Monate eine Therapie mit Etanercept erhielten, in die prospektive Kohortenstudie eingeschlossen. Der Psoriasis Area and Severity Index (PASI) wurde zu Monat $\mathrm{O}(\mathrm{MO}), \mathrm{M1}, \mathrm{M} 3$ und $\mathrm{M} 6$ bestimmt und die entsprechende PASI-75/90-Antwort ausgewertet. Bei jeder Visite wurden zudem Angst- und Depressionssymptome mithilfe der Hospital Anxiety and Depression Scale (HADS) beurteilt.

Unter der Behandlung mit Etanercept reduzierten sich im Vergleich zu MO die HADS-Depression (HADS-D)- und HADS-Ängstlichkeit (HADS-A)-Werte statistisch signifikant.

Das zentrale Ergebnis der Studie war, dass Patienten, die zu Beginn der Untersuchung depressiv waren, zu M3 und M6 eine geringere PASI-75-Antwort und zu M6 eine verringerte PASI-90-Antwort im Vergleich zu Patienten aufwiesen, die zu Beginn der Untersuchung nicht depressiv waren.

Darüber hinaus identifizierten multivariate logistische Regressionsanalysen Depressivität zu Studienbeginn als unabhängigen prädiktiven Faktor für eine geringere Ansprechwahrscheinlichkeit in Bezug auf eine PASI-75- und PASI-90-Antwort zu M6. Es wurde jedoch keine Korrelation zwischen der Ängstlichkeit bei Studienbeginn und der PASI-75/90-Antwort beobachtet.

\section{Kritik und Fazit für die Praxis}

Die vorgestellte prospektive Kohortenstudie von Jin et al. zeigt, dass das Vorhandensein von depressiven Symptomen zu Beginn einer antipsoriatischen Therapie das Therapieansprechen auf Etanercept negativ beeinflussen kann.
Folgende Punkte sind jedoch kritisch anzumerken: Zum einen wurde die Studie an einem relativ kleinen (chinesischen) Patientenkollektiv (85 Patienten), über eine relativ kurze Zeit (6 Monate) und nur für eine therapeutische Substanz (Etanercept) durchgeführt. Zum anderen fällt auf, dass in der Gruppe der depressiven Patienten ( $n=$ 35) der Anteil der weiblichen Patienten deutlich höher war (54,3\%) als in der Gruppe der nicht depressiven Patienten (24\%). Zudem war der mediane PASI zu Beginn der Studie in der Gruppe der depressiven Patienten höher als in der Gruppe der nicht depressiven Patienten (18,2 vs. 10,5). Des Weiteren wurde das Vorhandensein einer Depression nur anhand der Beantwortung des HADS-Fragebogens festgemacht und nicht durch einen Psychiater verifiziert.

Auch wenn es einige Kritikpunkte an der vorgestellten Studie gibt, ist es sinnvoll, das Ergebnis zu nutzen, um dafür zu sensibilisieren, dass psychiatrische Komorbiditäten von Psoriasis-Patienten engmaschig beobachtet werden sollten.

Die Konsensusempfehlung der Nationalen Konferenz zur Versorgung der Psoriasis empfiehlt, zur Früherkennung der Komorbidität «Depression» bei Psoriasis den «Zwei-Fragen-Test» zur Erfassung depressiver Symptome bei leichter Psoriasis alle 12 Monate und bei schwerer, inklusive systemtherapierter Psoriasis alle 6 Monate durchzuführen. Die Fragen, die beim Zwei-Fragen-Test gestellt werden, sind: «Fühlten Sie sich im letzten Monat häufig niedergeschlagen, traurig, bedrückt oder hoffnungslos?» und «Hatten Sie im letzten Monat deutlich weniger Lust und Freude an Dingen, die Sie sonst gerne tun?» Werden beide Fragen mit «ja» beantwortet, sollten die Patienten einer entsprechenden Therapie zugeführt werden [7].

Bei bestehender psychiatrischer Komorbidität sollte ein besonderes Augenmerk darauf gerichtet werden, dass die Psoriasis optimal behandelt und kontrolliert wird, um die psychische Belastung nicht noch durch eine fortwährende Stigmatisierung zu erhöhen.

\section{Disclosure Statement}

Hiermit erkläre ich, dass keine Interessenskonflikte in Bezug auf den vorliegenden Wissenstransfer bestehen.

\section{Literatur}

1 Koo J, Marangell LB, Nakamura M, et al. J Eur Acad Dermatol Venereol 2017: 31:1999-2009.

- 2 Tee SI, Lim ZV, Theng CT, et al. J Eur Acad Dermatol Venereol 2016;30: 1159-1164.

3 Dauden E, Castaneda S, Suarez C, et al. J Eur Acad Dermatol Venereol 2013; 27:1387-1404.

4 Connor CJ, Liu V, Fiedorowicz JG. Dermatol Res Pract 2015;2015:409637.

5 Wu JJ, Feldman SR, Koo J, et al. J Dermatol Treat 2018;29:487-495.

-6 Patel ZS, Hoffman LK, Buse DC, Grinberg AS, Afifi L, Cohen SR, et al. Curr Pain Headache Rep 2017;21:49.

7 Radtke MA, Mrowietz U, Feuerhahn J, et al. J Dtsch Dermatol Ges 2015;13: 674-690.

Kontaktadresse: Dr. Wiebke Sondermann, Universitätsklinikum Essen, Klinik für Dermatologie, Venerologie und Allergologie, Hufelandstraße 55, 45147 Essen, Deutschland, wiebke.sondermann@uk-essen.de 


\section{DuEPublico}

Duisburg-Essen Publications online

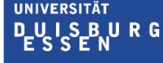

offen im Denken

$\mathbf{U b} \mid$\begin{tabular}{l|l} 
universitäts \\
bibliothek
\end{tabular}

Dieser Text wird via DuEPublico, dem Dokumenten- und Publikationsserver der Universität Duisburg-Essen, zur Verfügung gestellt. Die hier veröffentlichte Version der E-Publikation kann von einer eventuell ebenfalls veröffentlichten Verlagsversion abweichen.

DOI: $\quad 10.1159 / 000496272$

URN: urn:nbn:de:hbz:464-20220217-093757-0

Dieser Beitrag ist mit Zustimmung des Rechteinhabers aufgrund einer (DFG-geförderten) Allianz- bzw. Nationallizenz frei zugänglich.

Alle Rechte vorbehalten. 